TRANSACTIONS OF THE

AMERICAN MATHEMATICAL SOCIETY

Volume 361, Number 10, October 2009, Pages 5263-5283

S 0002-9947(09)04751-5

Article electronically published on April 8, 2009

\title{
VIRTUAL YANG-BAXTER COCYCLE INVARIANTS
}

\author{
JOSE CENICEROS AND SAM NELSON
}

\begin{abstract}
We extend the Yang-Baxter cocycle invariants for virtual knots by augmenting Yang-Baxter 2-cocycles with cocycles from a cohomology theory associated to a virtual biquandle structure. These invariants coincide with the classical Yang-Baxter cocycle invariants for classical knots but provide extra information about virtual knots and links. In particular, they provide a method for detecting non-classicality of virtual knots and links.
\end{abstract}

\section{INTRODUCTION}

In [5], quandle 2-cocycle invariants are generalized to the biquandle case. These are link invariants associated to finite biquandles and 2-cocycles in their YangBaxter cohomology. In [14, biquandles are generalized to include an operation at virtual crossings, a structure called a virtual biquandle.

A virtual link is an equivalence class of link diagrams with classical crossings and virtual crossings under virtual Reidemeister moves. Virtual crossings are crossings which arise from the genus in the surface on which the knot diagram is drawn, as distinguished from ordinary classical crossings.

Biquandles are algebras with axioms derived from the Reidemeister moves. Associated to a finite biquandle $T$ is the counting invariant $|\operatorname{Hom}(B(L), T)|$, the number of colorings of a link diagram by $T$. The Yang-Baxter 2-cocycle invariants are jazzed-up versions of this counting invariant, where we add up the values of a function $\phi$ at every crossing in each biquandle-colored diagram to obtain a Boltzmann weight for that coloring. Such a function $\phi$ defines an invariant provided $\phi$ is a Yang-Baxter 2-cocycle.

Including a virtual operation in a finite biquandle structure strengthens the counting invariants for virtual links. In this paper we describe a new method for strengthening and extracting more information from the virtual biquandle counting invariants using Yang-Baxter cocycles together with cocycles from a cohomology theory associated to the virtual operation. In particular, the new invariants can detect non-classicality in some virtual links.

Received by the editors September 6, 2007.

2000 Mathematics Subject Classification. Primary 57M27, 18G60.

(C)2009 American Mathematical Society

Reverts to public domain 28 years from publication 
The paper is organized as follows. In section 2 we recall the basics of virtual knot theory; in section 3 we recall virtual quandles and virtual biquandles. In section 4 we recall Yang-Baxter cohomology. In section 5 we introduce $S$-homology and our virtual Yang-Baxter cocycle invariants. In section 6 we see some examples of the new invariants, and in section 7 we collect some questions for future research.

\section{Classical and Virtual KnOts AND LinKS}

A classical oriented link diagram is a planar 4-valent directed graph with two edges directed in and two directed out at every vertex. The vertices are regarded as crossings, so one inbound/outbound pair forms an over-crossing strand and the other pair forms an under-crossing strand; we indicate which is which by drawing the under-crossing strand as a broken strand. It is well known that two classical oriented link diagrams represent ambient isotopic links if and only if they are related by a finite sequence of oriented Reidemeister moves. Here we depict the unoriented moves:
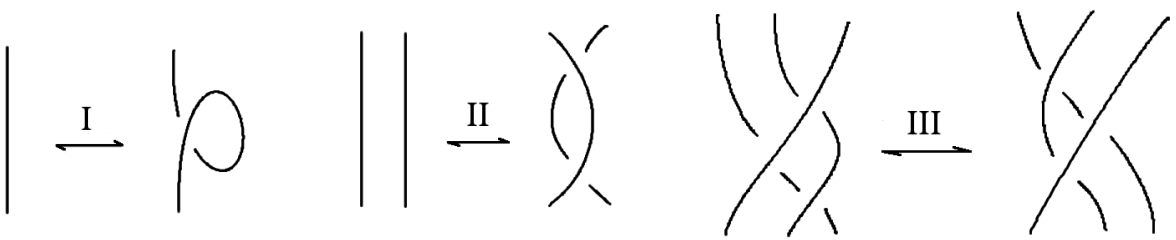

A virtual oriented link diagram is an oriented link diagram in which we drop the planarity requirement. We may draw such a diagram on an orientable surface $\Sigma$ with genus $g \geq 0$, and the represented virtual link is really an ordinary link whose ambient space is $\Sigma \times[0,1]$. Classical links form a subset of virtual links. To draw an oriented virtual link on planar paper, we must distinguish crossings arising from the genus in $\Sigma$ from ordinary classical crossings (decorated vertices in the non-planar graph); the former are called virtual crossings, represented as circled self-intersections with no over/under sense. Virtual links were introduced in [12] and have been studied in many recent works.

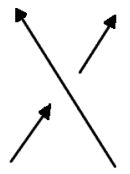

$-$
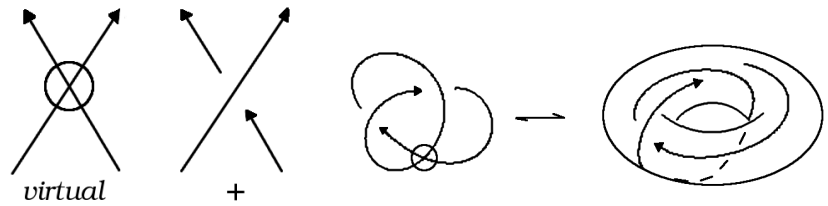

We may also represent an oriented link diagram using a signed Gauss code in which every classical crossing is assigned a label and a plus or minus sign according to its local writhe number or crossing sign. A signed Gauss code is then the result of traveling around each component of the oriented link diagram in the direction indicated by the orientation and noting the order in which each over-crossing and under-crossing is encountered. 
Example 2.1. The figure eight knot and its signed Gauss code.

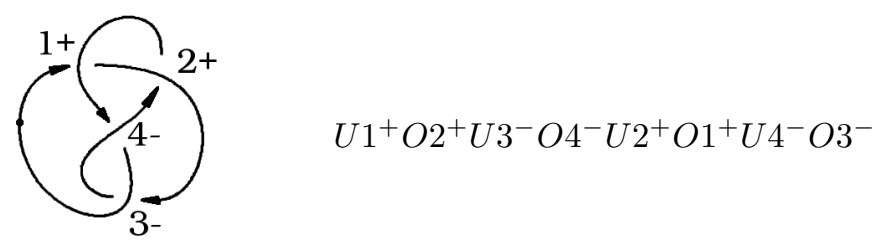

Indeed, signed Gauss codes provide one motivation for studying virtual links: while not every Gauss code corresponds to a planar link diagram, every signed Gauss code can be interpreted as a virtual link diagram by simply inserting virtual crossings as needed while drawing the link. A virtual link is then an equivalence class of oriented virtual link diagrams or, equivalently, signed Gauss codes, under the equivalence relation generated by the three Reidemeister moves.

Since virtual crossings are not included in ordinary signed Gauss codes, any two oriented virtual link diagrams with the same signed Gauss code are equivalent. Such diagrams are related by the detour move, which can be broken down into four virtual Reidemeister moves.
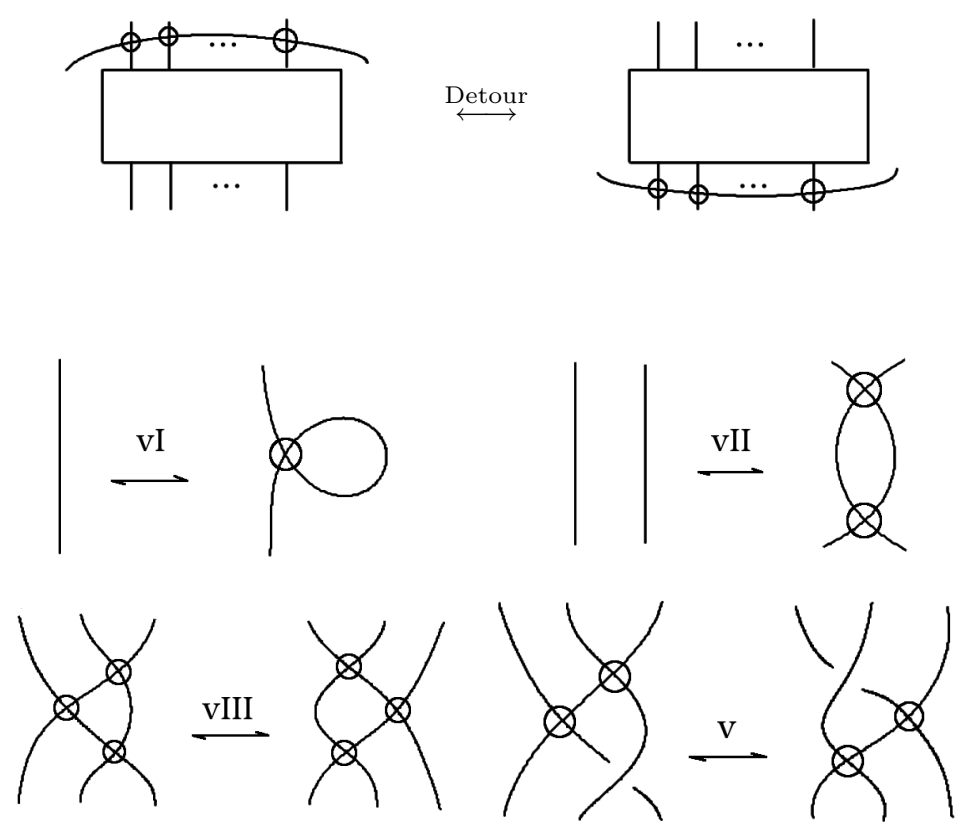

We may then consider virtual knots to be equivalence classes of planar virtual knot diagrams under the equivalence relation generated by the three Reidemeister moves and the detour move, or equivalently, the three Reidemeister moves and the four virtual moves $v, v I, v I I$ and $v I I I$. Note that while move $v$ permits a strand with only virtual crossings to be moved past a classical crossing, we cannot move a strand with classical crossings over or under a virtual crossing, since this changes the Gauss code and, generally, the virtual knot type. 
Virtual crossings are not usually included in a signed Gauss code, since in one sense the whole point of virtual crossings is to avoid testing codes for planarity. However, to compute the new invariants that we will define in section [6, we will need to specify the location of our virtual crossings. Thus, we have

Definition 2.2. A virtual signed Gauss code is a signed Gauss code in which we specify the virtual crossings in addition to the classical crossings, with " $R$ " and " $L$ " labels indicating whether we are entering the virtual crossing from the right or left input.

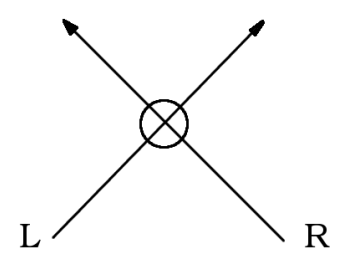

Example 2.3. A virtual knot diagram and its virtual signed Gauss code.

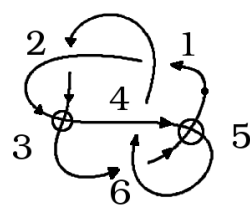

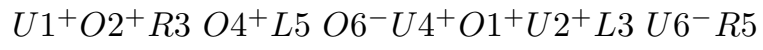

The following observation will be useful.

Lemma 2.4. The classical and virtual II moves, together with one oriented $v$ or vIII move, imply the other oriented $v$ and vIII moves. That is, we can reverse the direction of any strand in a type $v$ or vIII move using a sequence of II and vII moves.

Proof. We illustrate the case of reversing the virtual strand in a $\mathrm{v}$ move using virtual II moves. The other cases are similar.

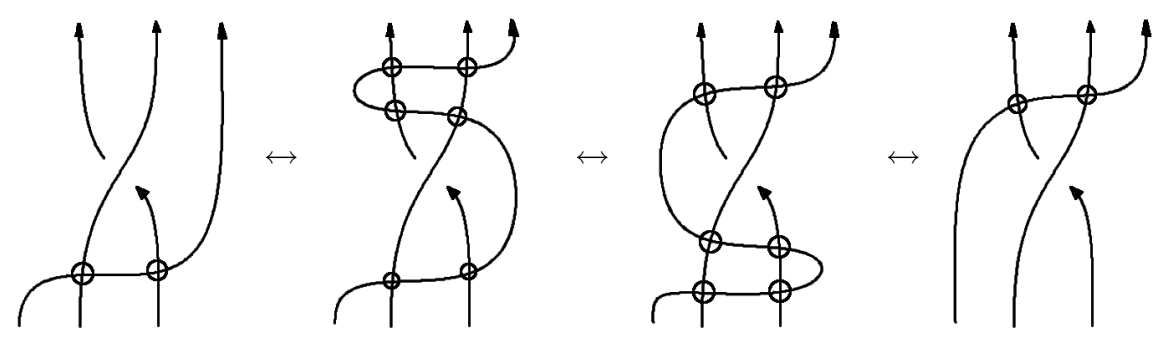




\section{Virtual QuANDles AND Biquandles}

The combinatorial approach to knot theory described in the previous section has a corresponding algebraic approach, which we now describe.

Definition 3.1. A biquandle is a set $B$ with four binary operations denoted $(a, b) \mapsto a^{b}, a^{\bar{b}}, a_{b}, a_{\bar{b}}$ satisfying the following axioms:

1. For every $a, b \in B$ we have

$$
a=\left(a^{b}\right)^{\left(\overline{b_{a}}\right)}, \quad b=\left(b_{a}\right)_{\left(\overline{a^{b}}\right)}, \quad a=\left(a^{\bar{b}}\right)^{\left(b_{\bar{a}}\right)}, \quad \text { and } \quad b=\left(b_{\bar{a}}\right)_{\left(a^{\bar{b}}\right)} .
$$

2. For every $a, b \in B$ there exist unique $x, y \in B$ such that

$$
x=a^{\left(b_{\bar{x}}\right)}, \quad a=x^{\bar{b}}, \quad b=\left(b_{\bar{x}}\right)_{a}, \quad y=a^{\left(\overline{b_{y}}\right)}, \quad a=y^{b}, \quad \text { and } \quad b=\left(b_{y}\right)_{\bar{a}} .
$$

3. For every $a, b, c \in B$ we have

$$
\begin{aligned}
& \left(a^{b}\right)^{c}=\left(a^{\left(c_{b}\right)}\right)^{\left(b^{c}\right)}, \quad\left(c_{b}\right)_{a}=\left(c_{\left(a_{b}\right)}\right)_{\left(b_{a}\right)}, \quad\left(b_{a}\right)^{\left(c_{\left(a^{b}\right)}\right)}=\left(b^{c}\right)_{\left(a^{\left(c_{b}\right)}\right)},
\end{aligned}
$$

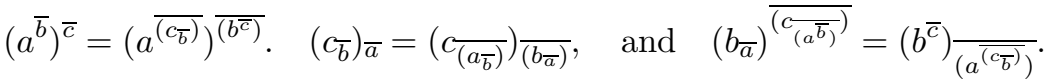

4. For every $a \in B$ there exist unique $x, y \in B$ such that

$$
x=a_{x}, \quad a=x^{a}, \quad y=a^{\bar{y}} \text { and } \quad a=y_{\bar{a}} .
$$

A biquandle in which $a_{b}=a_{\bar{b}}=a$ for all $a, b \in B$ is a quandle; if $B$ is a quandle, we may denote $a^{b}=a \triangleright b$ and $a^{\bar{b}}=a \triangleright^{-1} b$.

The biquandle axioms are obtained by associating elements of $B$ to semiarcs (i.e., edges in the link diagram considered as a 4-valent graph) in an oriented link diagram and letting these elements act on each other at crossings as pictured. The notation is chosen so that $a^{b}$ suggests " $a$ under $b$ " and $b_{a}$ suggests " $b$ over $a$."
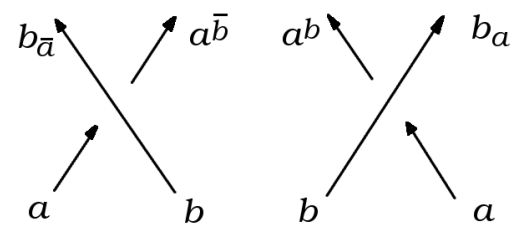

The set of biquandle axioms is then the result of checking the conditions imposed by a minimal set of oriented Reidemeister moves. For example, the type II Reidemeister move with both strands oriented in the same direction (the direct II-move) implies axiom (1) above:
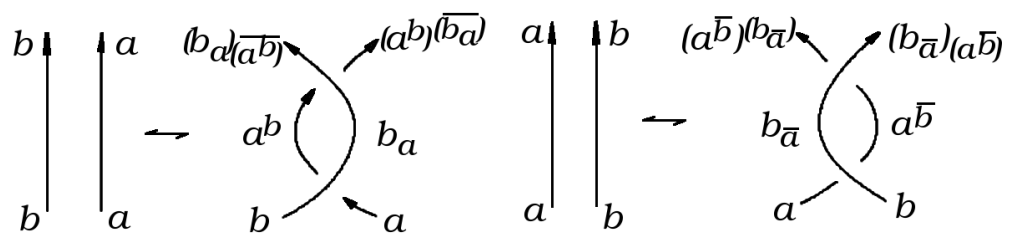

See [13] for more. 
Biquandles are useful for defining invariants of knots and links. Specifically, for every knot or link diagram there is an associated knot biquandle defined as the quotient of the free biquandle on the set of semiarcs in the diagram by the equivalence relation generated by the relations determined by the crossings in the diagram.

Given a finite biquandle $T$, we can obtain an easily computable link invariant by computing the cardinality $|\operatorname{Hom}(B(L), T)|$ of the set of biquandle homomorphisms from the knot biquandle $B(L)$ of the link $L$ into $T$. Each such homomorphism can be pictured as a "coloring" of $L$ which associates an element of $T$ to each semiarc in $L$ such that the biquandle operations in $T$ are compatible with the relations determined by the crossings in $L$.

Thus, for every finite biquandle $T$ there is a counting invariant $|\operatorname{Hom}(B(L), T)|$. Several recent papers describe examples of finite biquandles (see [6], 8] and [15] for example). One nice example is the family of Alexander biquandles, where $T$ is a module over the ring $\mathbb{Z}\left[s^{ \pm 1}, t^{ \pm 1}\right]$ of two-variable Laurent polynomials with integer coefficients. Such a $T$ is a biquandle with operations

$$
a^{b}=t a+(1-s t) b, a^{\bar{b}}=t^{-1} a+\left(1-s^{-1} t^{-1}\right) b, a_{b}=s a, a_{\bar{b}}=s^{-1} a .
$$

For example, for any $n \geq 2$ we can get an Alexander biquandle of cardinality $n$ by taking $T=\mathbb{Z}_{n}$ and choosing $s, t$ to be invertible elements of $\mathbb{Z}_{n}$.

Example 3.2. The trefoil knot $3_{1}$ has three colorings by the Alexander biquandle $\mathbb{Z}_{3}$ with $s=t=2$ :
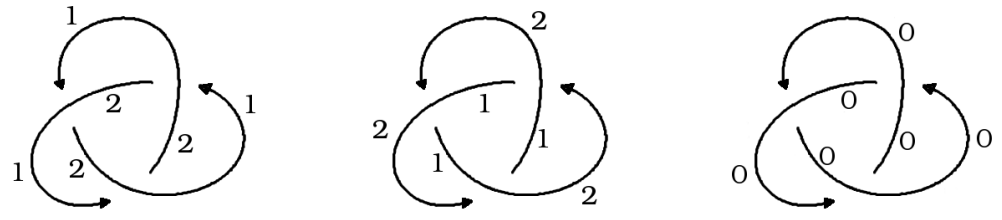

Hence, we have $\left|\operatorname{Hom}\left(B\left(3_{1}\right), T\right)\right|=3$.

We can specify a finite biquandle $T=\left\{x_{1}, \ldots, x_{n}\right\}$ by listing its operation tables in the form of a block matrix $M_{T}=\left[\begin{array}{c|c}i^{j} & i^{j} \\ \hline i_{\bar{j}} & i_{j}\end{array}\right]$; for example, the biquandle $T=\mathbb{Z}_{3}$ with $s=t=2$ from Example 3.2 has biquandle matrix

$$
M_{T}=\left[\begin{array}{lll|lll}
2 & 2 & 2 & 2 & 2 & 2 \\
1 & 1 & 1 & 1 & 1 & 1 \\
3 & 3 & 3 & 3 & 3 & 3 \\
\hline 2 & 2 & 2 & 2 & 2 & 2 \\
1 & 1 & 1 & 1 & 1 & 1 \\
3 & 3 & 3 & 3 & 3 & 3
\end{array}\right]
$$

where we use $x_{1}=[1], x_{2}=[2]$ and $x_{3}=[0]$. This notation has the advantage of letting us compute the counting invariant even in the absence of a convenient algebraic description of the structure of $T$. See [16] and [7] for more.

For virtual knots, we can simply ignore the presence of virtual crossings and compute the counting invariant with respect to a finite biquandle $T$, since the 
Reidemeister moves are local and hence agnostic of the genus of the surface on which $L$ is drawn.

An alternative idea is to consider virtual crossings in our algebraic structure, dividing our virtual link diagrams at both classical and virtual crossings and introducing an additional operation at the virtual crossings. This approach was taken in [14]; the resulting algebraic structure is called a virtual biquandle.

Definition 3.3. Let $B$ be a biquandle and let $S: B \rightarrow B$ be an automorphism of $B$; that is, $S$ is a bijection such that for all $a, b \in B$,

$$
S\left(a^{b}\right)=S(a)^{S(b)}, S\left(a_{b}\right)=S(a)_{S(b)}, S\left(a^{\bar{b}}\right)=S(a)^{\overline{S(b)}} \text {, and } S\left(a_{\bar{b}}\right)=S(a) \overline{S(b)} .
$$

Then the pair $(B, S)$ is a virtual biquandle. If $a_{b}=a_{\bar{b}}=a$ for all $a \in B$, then $B$ is a quandle and $(B, S)$ is a virtual quandle.

At a virtual crossing, the semiarc entering from the right picks up an $S$ while the semiarc entering from the left picks up an $S^{-1}$ :

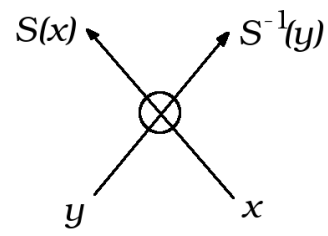

The pure virtual moves $v I$ and $v I I$ require that $S$ is bijective, move $v I I I$ imposes no conditions, and the oriented $v$ moves require that $S$ is an automorphism of $B$; see 14] (or, check it as an exercise!).

We may regard ordinary biquandles as the special case $S=I d_{B}$, so the theory of biquandles is a subset of the theory of virtual biquandles. Given a virtual link diagram $L$, one defines the knot virtual biquandle $V B(L)$ of $L$ as the quotient of the free virtual biquandle on the set of semiarcs in the virtual link diagram $L$ by the equivalence relation generated by the relations at the crossings in $L$. As before, we can regard a homomorphism $f: V B(L) \rightarrow(T, S)$ from the knot virtual biquandle of $L$ to a finite virtual biquandle $(T, S)$ as a coloring of $L$, i.e., an assignment of elements of $T$ to semiarcs in $L$ (divided at both classical and virtual crossings) such that the virtual biquandle operations are satisfied at every crossing.

Proposition 3.4. Let $B$ be a biquandle and $S, S^{\prime} \in \operatorname{Aut}(B)$. Then the two virtual biquandle structures $(B, S)$ and $\left(B, S^{\prime}\right)$ are isomorphic if and only if $S$ is conjugate to $S^{\prime}$.

Proof. An isomorphism of virtual biquandles $\phi:(B, S) \rightarrow\left(B, S^{\prime}\right)$ is an automorphism $\phi: B \rightarrow B$ such that for all $x \in B$ we have $\phi(S(x))=S^{\prime}(\phi(x))$, that is, $\phi S=S^{\prime} \phi$ or $S=\phi^{-1} S^{\prime} \phi$. Conversely, if $S=\phi^{-1} S^{\prime} \phi$ in $\operatorname{Aut}(B)$, then $\phi(S(x))=$ $S^{\prime}(\phi(x))$ for all $x \in B$ and $\phi$ is an isomorphism from $(B, S)$ to $\left(B, S^{\prime}\right)$.

Corollary 3.5. Given a biquandle B, we may list all virtual biquandle structures on $B$ by listing one representative from each conjugacy class of $\operatorname{Aut}(B)$. 
Example 3.6. The Alexander biquandle $T=\mathbb{Z}_{3}$ with $s=1, t=2$ has biquandle matrix

$$
M_{T}=\left[\begin{array}{lll|lll}
1 & 3 & 2 & 1 & 3 & 2 \\
3 & 2 & 1 & 3 & 2 & 1 \\
2 & 1 & 3 & 2 & 1 & 3 \\
\hline 1 & 1 & 1 & 1 & 1 & 1 \\
2 & 2 & 2 & 2 & 2 & 2 \\
3 & 3 & 3 & 3 & 3 & 3
\end{array}\right] .
$$

In fact, this is an Alexander quandle, $\mathbb{Z}\left[t^{ \pm 1}\right] /(t-2)$. It has automorphism group $S_{3}$, with conjugacy classes represented by (1), (23) and (123). Hence, up to isomorphism, there are three virtual quandle structures on $T$.

If a virtual diagram $L$ represents a classical knot or link, then the counting invariants with respect to any two virtual biquandle structures on a given biquandle must be equal since both can be obtained from a diagram equivalent to $L$ with no virtual crossings. Thus we have:

Proposition 3.7. If $|\operatorname{Hom}(V B(L),(T, S))| \neq\left|\operatorname{Hom}\left(V B(L),\left(T, S^{\prime}\right)\right)\right|$ for any two virtual biquandle structures $(T, S)$ and $\left(T, S^{\prime}\right)$ defined on the same biquandle $T$, then $L$ is non-classical.

Example 3.8. The virtual quandle structures on the Alexander quandle $T=$ $\mathbb{Z}\left[t^{ \pm 1}\right] /(t-2)$ show that the virtual trefoil is non-classical, since $(T,(1))$ yields a counting invariant value of 3 while $(T,(123))$ has a counting invariant value of 0 .

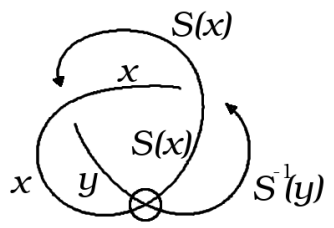

$$
\begin{aligned}
2 S^{-1}(y)+2 S(x) & =x \\
2 S(x)+2 x & =y
\end{aligned}
$$

Here we have $x^{y}=x^{\bar{y}}=2 x+2 y(\bmod 3)$ and $x_{y}=x_{\bar{y}}=x$. Thus, colorings are solutions in $\mathbb{Z}_{3}$ to the above system of equations. If $S(x)=x$, the system reduces to $\begin{aligned} & y=x \\ & x=y\end{aligned}$ and there is one solution for every element of $T$. On the other hand, the permutation $S=(123)$ translates as $S(x)=x+1$, and our system becomes

$$
\begin{aligned}
2 y-2+2 x+2 & =x \\
2 x+2+2 x & =y
\end{aligned} \quad \text { which reduces to } \quad \begin{aligned}
y & =x \\
x+2 & =y
\end{aligned}
$$

which is inconsistent.

The counting invariants associated to virtual biquandles provide a sharpening and strengthening of the biquandle counting invariants for virtual knots and links, while they coincide with the usual biquandle counting invariants for classical knots and links. In the next section, we will see a method for further improving and strengthening these invariants.

\section{YANG-BAXTer COHOMOLOGY}

The counting invariant $|\operatorname{Hom}(B(L), T)|$ associated to a finite biquandle $T$ is a computable and useful invariant of knots and links. However, a set is more than 
a mere cardinality, and it is natural to seek ways of extracting more information from the set $\operatorname{Hom}(B(L), T)$.

One very successful idea, with origins in statistical mechanics, is to associate to every crossing a function $\phi$ with values in some abelian group; then for a given element $f \in \operatorname{Hom}(B(L), T)$, we can sum our values of $\phi$ over the crossings in the diagram colored by $f$ to obtain a Boltzmann weight for $f$. The set-with-multiplicities of all such weights for all $f \in \operatorname{Hom}(B(L), T)$ is then an invariant of knots and links provided the function $\phi$ is chosen such that the Boltzmann weight for each colored diagram is unchanged by Reidemeister moves.

The condition on $\phi$ required to make this happen turns out to be expressible in terms of a cohomology theory associated to the coloring quandle or biquandle $T$. Many variations of this idea exist, including the quandle homology described in [3], the twisted quandle homology described in 4], the quandle homology with coefficients in a quandle module ([1], [10]), the biquandle version known as YangBaxter homology ([5]), and a unifying approach to the homology of quandles and Lie algebras $([2])$.

Since our focus is on virtual biquandles, we will use the Yang-Baxter homology approach from 5. Moreover, we will choose our coefficient ring to be $\mathbb{Q}$ for simplicity of computation. Then if $B$ is a biquandle, a function $\phi: B \times B \rightarrow \mathbb{Q}$ is a Yang-Baxter 2-cocycle if $\phi$ satisfies

$$
\phi(a, b)+\phi\left(a^{b}, c\right)+\phi\left(b_{a}, c_{a^{b}}\right)=\phi(b, c)+\phi\left(a, c_{b}\right)+\phi\left(a^{c_{b}}, b^{c}\right)
$$

for all $a, b, c \in B$. If $\phi(x, a)=\phi(a, y)=0$ for all $a \in B$, where $x, y$ are the elements of $B$ from biquandle axiom (4), then $\phi$ is a reduced Yang-Baxter 2-cocycle. We will refer to such cocycles as classical cocycles.

As implied by the term "2-cocycle", these functions $\phi$ are elements of the second cohomology in a cohomology theory, described in [5]. The chain groups are generated by ordered $n$-tuples of biquandle elements, and the boundary map is obtained by interpreting an $n$-tuple as colors on a preferred path in the $n$-cube from $(0,0, \ldots, 0)$ to $(1,1, \ldots, 1)$. Such a coloring then extends in a unique way to the whole $n$-cube graph; one then takes the usual boundary of the $n$-cube and interprets the faces as the $(n-1)$-tuples corresponding to their preferred paths. It is perhaps more enlightening, however, to consider the Reidemeister III move:
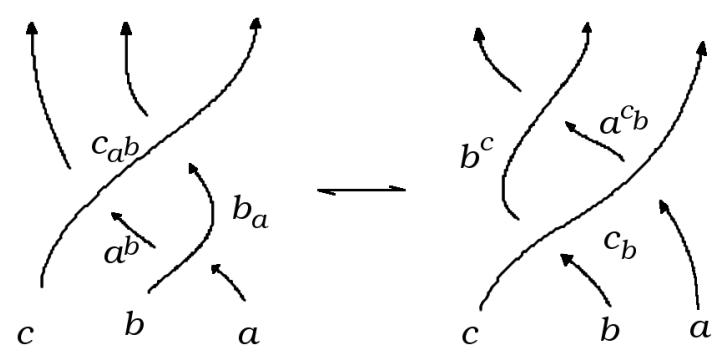
Here the rule is that every positive crossing contributes $\phi(u, o)$, where $u$ is the color on the under-crossing inbound semiarc and $o$ is the color on the over-crossing inbound semiarc, while negative crossings contribute $-\phi(u, o)$, where $u$ is the undercrossing outbound semiarc, and $o$ is the over-crossing outbound semiarc.
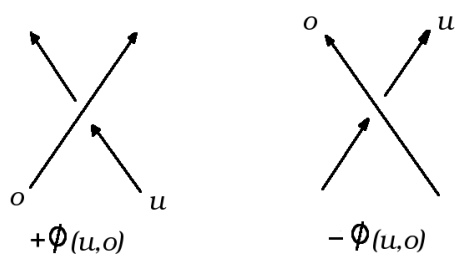

This rule guarantees that the Boltzmann weights are unchanged by Reidemeister II moves; the cocycle condition takes care of the type III moves and the reduced condition takes care of the type I moves. The classical Yang-Baxter 2-cocycle invariant is then

$$
\Phi_{Y B}(L)=\sum_{f \in \operatorname{Hom}(B(L), T)} t^{B W(f)}
$$

where $B W(f)=\sum_{\text {crossings }} \pm \phi(u, o)$ is the Boltzmann weight of the homomorphism $f$.

Finally, it is observed in [5] that if $f=\delta_{Y B}^{1}(g)=g\left(\partial_{2}^{Y B}\right)$ is a Yang-Baxter coboundary, then at every crossing $f$ effectively counts $g(u i)+g(o i)-g(u o)-g(o o)$ where $u i$, oi, uo and oo are the inbound under-crossing, inbound over-crossing, outbound under-crossing, and outbound over-crossing semiarcs respectively. Then since every semiarc appears in the sum once as an inbound and once as an outbound semiarc, the total Boltzmann weight of a coboundary is zero and cohomologous cocycles define the same invariant.

\section{5. $S$-HOMOLOGY}

For virtual biquandles $(B, S)$, we now define a second cohomology theory which we will use to augment the classical theory for defining invariants of virtual knots and links. First, we need a rule for contributions to the Boltzmann weight from each virtual crossing in a virtual biquandle colored diagram. Let $v: B \times B \rightarrow \mathbb{Q}$; then at each virtual crossing, we will count $v(x, y)-v\left(S^{-1}(y), S(x)\right)$ where $x$ is the biquandle color on the right-hand input semiarc, $y$ is the color on the left-hand input semiarc, $S^{-1}(y)$ is the output right-hand semiarc and $S(x)$ is the color on the left-hand output semiarc.

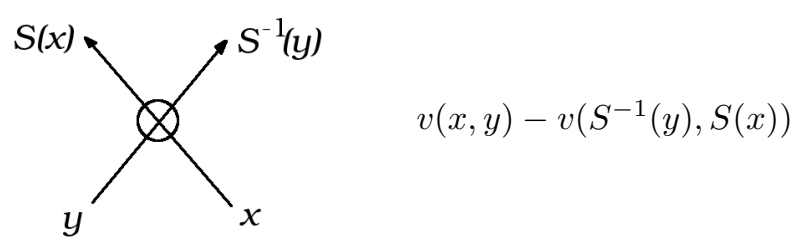

Proposition 5.1. The contribution to the Boltzmann weight of a colored virtual link diagram with the above rule is unchanged by vI and vII moves. 
Proof. Move $v I$ :

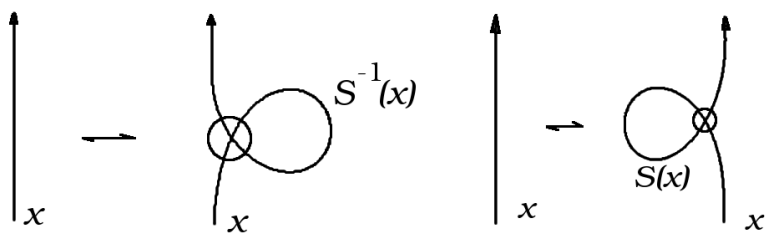

In the first move, the contribution to the Boltzmann weight on the left is 0 , and on the right, the contribution is $v\left(S^{-1}(x), x\right)-v\left(S^{-1}(x), x\right)=0$. In the second move, on the left again we have a contribution of 0 and on the right we have $v(x, S(x))-v(x, S(x))=0$.

Move vII: There are two cases, one with the two strands oriented in the same direction and one with the two strands oriented in opposite directions.

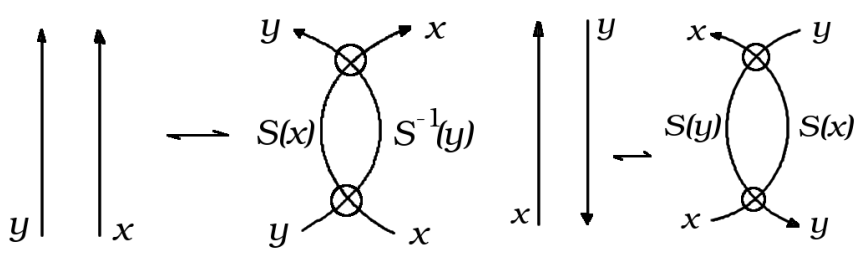

Here, in the first move the contribution on the left is again 0 , while on the right we have a contribution to the Boltzmann weight of $v(x, y)-v\left(S^{-1}(y), S(x)\right)+$ $v\left(S^{-1}(y), S(x)\right)-v(x, y)=0$. Similarly, in the second move the contribution on the left is 0 while the contribution on the right is $v(x, S(y))-v(y, S(x))+v(y, S(x))-$ $v(x, S(y))=0$.

To deal with the virtual type III move, we now define a chain complex associated to the virtual biquandle operation $S: B \rightarrow B$. As in the classical case, we first let $C_{n}(B, S ; \mathbb{Q})=\mathbb{Q}\left[B^{n}\right]$, the rational vector space with basis consisting of all $n$-tuples of elements of the coloring biquandle $B$. Let

$$
\partial_{i}^{1}\left(x_{1}, \ldots, x_{n}\right)=\left(S\left(x_{1}\right), \ldots, S\left(x_{i-1}\right), x_{i+1}, \ldots, x_{n}\right)
$$

and

$$
\partial_{i}^{2}\left(x_{1}, \ldots, x_{n}\right)=\left(x_{1}, \ldots, x_{i-1}, S^{-1}\left(x_{i+1}\right), \ldots, S^{-1}\left(x_{n}\right)\right) .
$$

Let us abbreviate $\mathbf{x}=\left(x_{1}, \ldots, x_{n}\right)$. Then let

$$
\partial_{n}^{S}(\mathbf{x})=\sum_{i=1}^{n}(-1)^{i}\left(\partial_{i}^{1}(\mathbf{x})-\partial_{i}^{2}(\mathbf{x})\right)
$$

and extend $\partial_{n}^{S}$ to $C_{n}(B, S ; \mathbb{Q})$ by linearity.

Proposition 5.2. With the above definition, $\left(C_{*}(B, S ; \mathbb{Q}), \partial_{*}^{S}\right)$ is a chain complex. 
Proof.

$$
\begin{aligned}
\partial_{n-1}^{S}\left(\partial_{n}^{S}(\mathbf{x})\right)= & \sum_{j=1}^{n-1} \sum_{i=1}^{n}(-1)^{i+j}\left(\partial_{j}^{1} \partial_{i}^{1}(\mathbf{x})-\partial_{j}^{1} \partial_{i}^{2}(\mathbf{x})-\partial_{j}^{2} \partial_{i}^{1}(\mathbf{x})+\partial_{j}^{2} \partial_{i}^{2}(\mathbf{x})\right) \\
= & \sum_{i>j}(-1)^{i+j}\left(\partial_{j}^{1} \partial_{i}^{1}(\mathbf{x})-\partial_{j}^{1} \partial_{i}^{2}(\mathbf{x})-\partial_{j}^{2} \partial_{i}^{1}(\mathbf{x})+\partial_{j}^{2} \partial_{i}^{2}(\mathbf{x})\right) \\
& -\sum_{i<j}(-1)^{i+j}\left(\partial_{j}^{1} \partial_{i}^{1}(\mathbf{x})-\partial_{j}^{1} \partial_{i}^{2}(\mathbf{x})-\partial_{j}^{2} \partial_{i}^{1}(\mathbf{x})+\partial_{j}^{2} \partial_{i}^{2}(\mathbf{x})\right) .
\end{aligned}
$$

Now, if $i>j$ we have

$$
\begin{aligned}
\partial_{j}^{1} \partial_{i}^{1}(\mathbf{x})= & \left(S^{2}\left(x_{1}\right), \ldots, S^{2}\left(x_{j-1}\right), S\left(x_{j+1}\right), \ldots, S\left(x_{i-1}\right), x_{i+1}, \ldots, x_{n}\right), \\
\partial_{j}^{1} \partial_{i}^{2}(\mathbf{x})= & \left(S\left(x_{1}\right), \ldots, S\left(x_{j-1}\right), x_{j+1}, \ldots, x_{i-1}, S^{-1}\left(x_{i+1}\right), \ldots, S^{-1}\left(x_{n}\right)\right), \\
\partial_{j}^{2} \partial_{i}^{1}(\mathbf{x})= & \left(S\left(x_{1}\right), \ldots, S\left(x_{j-1}\right),\right. \\
& \left.S^{-1}\left(S\left(x_{j+1}\right)\right), \ldots, S^{-1}\left(S\left(x_{i-1}\right)\right), S^{-1}\left(x_{i+1}\right), \ldots, S^{-1}\left(x_{n}\right)\right) \\
= & \left(S\left(x_{1}\right), \ldots, S\left(x_{j-1}\right), x_{j+1}, \ldots, x_{i-1}, S^{-1}\left(x_{i+1}\right), \ldots, S^{-1}\left(x_{n}\right)\right), \\
\partial_{j}^{2} \partial_{i}^{2}(\mathbf{x})= & \left(x_{1}, \ldots, x_{j-1}, S^{-1}\left(x_{j+1}\right), \ldots, S^{-1}\left(x_{i-1}\right), S^{-2}\left(x_{i+1}\right), \ldots, S^{-2}\left(x_{n}\right)\right),
\end{aligned}
$$

and for $i<j$ we have

$$
\begin{aligned}
\partial_{j}^{1} \partial_{i}^{1}(\mathbf{x})= & \left(S^{2}\left(x_{1}\right), \ldots, S^{2}\left(x_{i-1}\right), S\left(x_{i+1}\right), \ldots, S\left(x_{j-1}\right), x_{j+1}, \ldots, x_{n}\right), \\
\partial_{j}^{1} \partial_{i}^{2}(\mathbf{x})= & \left(S\left(x_{1}\right), \ldots, S\left(x_{i-1}\right),\right. \\
& \left.S\left(S^{-1}\left(x_{i+1}\right)\right), \ldots, S\left(S^{-1}\left(x_{j-1}\right)\right), S^{-1}\left(x_{j+1}\right), \ldots, S^{-1}\left(x_{n}\right)\right) \\
= & \left(S\left(x_{1}\right), \ldots, S\left(x_{i-1}\right), x_{i+1}, \ldots, x_{j-1}, S^{-1}\left(x_{j+1}\right), \ldots, S^{-1}\left(x_{n}\right)\right), \\
\partial_{j}^{2} \partial_{i}^{1}(\mathbf{x})= & \left(S\left(x_{1}\right), \ldots, S\left(x_{i-1}\right), x_{i+1}, \ldots, x_{j-1}, S^{-1}\left(x_{j+1}\right), \ldots, S^{-1}\left(x_{n}\right)\right), \\
\partial_{j}^{2} \partial_{i}^{2}(\mathbf{x})= & \left(x_{1}, \ldots, x_{i-1}, S^{-1}\left(x_{i+1}\right), \ldots, S^{-1}\left(x_{j-1}\right), S^{-2}\left(x_{j+1}\right), \ldots, S^{-2}\left(x_{n}\right)\right) .
\end{aligned}
$$

Hence, the terms in the $i<j$ sum match up one-to-one with the terms in the $i>j$ sum, but with opposite signs, and we have $\partial_{n-1}^{S} \circ \partial_{n}^{S}=0$.

To find cocycles which satisfy the condition imposed by the virtual type III move, we need to restrict our attention to a certain subcomplex.

Definition 5.3. Let $\rho_{n}: B^{n} \rightarrow B^{n}$ be given by

$$
\rho_{n}\left(x_{1}, \ldots, x_{n}\right)=\left(S^{j_{1}}\left(x_{1}\right), \ldots, S^{j_{n}}\left(x_{n}\right)\right),
$$

where $j_{i}=n-2 i+1$, and let $\tau_{n}: B^{n} \rightarrow B^{n}$ be given by

$$
\tau_{n}\left(x_{1}, \ldots, x_{n}\right)=\left(x_{n}, \ldots, x_{1}\right) .
$$

Let $\omega_{n}: B^{n} \rightarrow B^{n}$ be given by $\omega_{n}(\mathbf{x})=\tau_{n}\left(\rho_{n}(\mathbf{x})\right)$.

The map $\omega_{n}$ reverses the order of the components of its argument and operates on each by a power of $S$ so that the sum of the power of $S$ and the subscript of $x$ 
equals the position in $\omega_{n}$ of each entry. That is, the $i$ th entry of $\omega_{n}\left(x_{1}, \ldots, x_{n}\right)$ is $S^{2 i-n-1}\left(x_{n-i+1}\right)$. For example, we have

$$
\omega_{4}\left(x_{1}, x_{2}, x_{3}, x_{4}\right)=\left(S^{-3}\left(x_{4}\right), S^{-1}\left(x_{3}\right), S\left(x_{2}\right), S^{3}\left(x_{1}\right)\right)
$$

and

$$
\omega_{3}\left(x_{1}, x_{2}, x_{3}\right)=\left(S^{-2}\left(x_{3}\right), x_{2}, S^{2}\left(x_{1}\right)\right) .
$$

Definition 5.4. Let $C_{n}^{S}(B, S ; \mathbb{Q})$ be the subspace of $C_{n}(B, S ; \mathbb{Q})$ generated by elements of the form

$$
\mathbf{x}+(-1)^{j} \omega_{n}(\mathbf{x}) \quad \text { where } \quad j=\left\{\begin{array}{cc}
0 & n \equiv 0,1 \bmod 4 \\
1 & n \equiv 2,3 \bmod 4 .
\end{array}\right.
$$

Lemma 5.5. For $* \in\{1,2\}$ we have $\omega_{n-1}\left(\partial_{i}^{*}(\mathbf{x})\right)=\partial_{n-i+1}^{*}\left(\omega_{n}(\mathbf{x})\right)$.

Proof. Let us denote $\left(y_{1}, \ldots, y_{n-1}\right)=\partial_{i}^{1}\left(x_{1}, \ldots, x_{n}\right)$; then we have $y_{j}=S\left(x_{j}\right)$ for $j<i$ and $y_{j}=x_{j+1}$ for $j>i$. Then

$$
\begin{aligned}
\omega_{n-1}\left(\partial_{i}^{1}(\mathbf{x})\right)= & \omega_{n-1}\left(y_{1}, \ldots, y_{i-1}, y_{i}, \ldots, y_{n-1}\right) \\
= & \tau_{n-1}\left(\rho_{n-1}\left(y_{1}, \ldots, y_{i-1}, y_{i}, \ldots, y_{n-1}\right)\right) \\
= & \tau_{n-1}\left(S^{(n-1)-1}\left(y_{1}\right), \ldots, S^{(n-1)-2(i-1)+1}\left(y_{i-1}\right)\right. \\
& \left.\quad S^{(n-1)-2 i+1}\left(y_{i}\right), \ldots, S^{1-(n-1)}\left(y_{n-1}\right)\right) \\
= & \tau_{n-1}\left(S^{n-2}\left(y_{1}\right), \ldots, S^{n-2 i+2}\left(y_{i-1}\right), S^{n-2 i}\left(y_{i}\right), \ldots, S^{-n}\left(y_{n-1}\right)\right) \\
= & \tau_{n-1}\left(S^{n-1}\left(x_{1}\right), \ldots, S^{n-2 i+3}\left(x_{i-1}\right), S^{n-2 i}\left(x_{i+1}\right), \ldots, S^{-n}\left(x_{n}\right)\right) \\
= & \left(S^{-n}\left(x_{n}\right), \ldots, S^{n-2 i}\left(x_{i+1}\right), S^{n-2 i+3}\left(x_{i-1}\right), \ldots, S^{n-1}\left(x_{1}\right)\right)
\end{aligned}
$$

while

$$
\begin{aligned}
\partial_{n-i+1}^{1}\left(\omega_{n}(\mathbf{x})\right)= & \partial_{n-i+1}^{1}\left(S^{1-n}\left(x_{n}\right), \ldots, S^{n-2 i-1}\left(x_{i-1}\right),\right. \\
& \left.S^{n-2 i+1}\left(x_{i}\right), S^{n-2 i+3}\left(x_{i+1}\right), \ldots, S^{1-n}\left(x_{1}\right)\right) .
\end{aligned}
$$

Here the entry $S^{n-2 i+1}\left(x_{i}\right)$ is in the $n-i+1$ position, so we have

$$
\begin{aligned}
\partial_{n-i+1}^{1}\left(\omega_{n}(\mathbf{x})\right)= & \left(S^{1-n+1}\left(x_{n}\right), \ldots, S^{n-2 i-1+1}\left(x_{i-1}\right)\right. \\
& \left.S^{n-2 i+3}\left(x_{i+1}\right), \ldots, S^{1-n}\left(x_{1}\right)\right) \\
= & \left(S^{-n}\left(x_{n}\right), \ldots, S^{n-2 i}\left(x_{i+1}\right)\right. \\
& \left.S^{n-2 i+3}\left(x_{i-1}\right), \ldots, S^{n-1}\left(x_{1}\right)\right)
\end{aligned}
$$

as required. The other case is similar.

Corollary 5.6. $\left(C_{n}^{S}(B ; \mathbb{Q}), \partial_{n}^{S}\right)$ is a subcomplex of $\left(C_{n}(B, S ; \mathbb{Q}), \partial_{n}^{S}\right)$. 
Proof. We must show that $\partial_{n}^{S}\left(C_{n}^{S}(B, S ; \mathbb{Q})\right) \subset C_{n-1}^{S}(B, S ; \mathbb{Q})$. For any generator $\mathbf{z}=\mathbf{x}+(-1)^{j} \omega_{n}(\mathbf{x}) \in C_{n}^{S}(B, S ; \mathbb{Q})$, we have

$$
\begin{aligned}
\partial_{n}^{S}(\mathbf{z})= & \partial_{n}^{S}\left(\mathbf{x}+(-1)^{j} \omega_{n}(\mathbf{x})\right) \\
= & \sum_{i=1}^{n}(-1)^{i}\left(\partial_{i}^{1}\left(\mathbf{x}+(-1)^{j} \omega_{n}(\mathbf{x})\right)-\partial_{i}^{2}\left(\mathbf{x}+(-1)^{j} \omega_{n}(\mathbf{x})\right)\right) \\
= & \sum_{i=1}^{n}(-1)^{i}\left(\partial_{i}^{1}(\mathbf{x})+(-1)^{j} \partial_{i}^{1} \omega_{n}(\mathbf{x})-\partial_{i}^{2}(\mathbf{x})+(-1)^{j+1} \partial_{i}^{2}\left(\omega_{n}(\mathbf{x})\right)\right) \\
= & \sum_{i=1}^{n}(-1)^{i}\left(\partial_{i}^{1}(\mathbf{x})+(-1)^{j} \omega_{n-1} \partial_{n-i+1}^{1}(\mathbf{x})-\partial_{i}^{2}(\mathbf{x})\right. \\
& \left.+(-1)^{j+1} \omega_{n-1} \partial_{n-i+1}^{2}(\mathbf{x})\right) \\
= & \sum_{i=1}^{n}(-1)^{i}\left(\partial_{i}^{1}(\mathbf{x})-\partial_{i}^{2}(\mathbf{x})\right) \\
& +(-1)^{j} \omega_{n-1}\left(\sum_{i=1}^{n}(-1)^{i}\left(\partial_{n-i+1}^{1}(\mathbf{x})-\partial_{n-i+1}^{2}(\mathbf{x})\right)\right) \\
= & \sum_{i=1}^{n}(-1)^{i}\left(\partial_{i}^{1}(\mathbf{x})-\partial_{i}^{2}(\mathbf{x})\right) \\
& +(-1)^{j+n+1} \omega_{n-1}\left(\sum_{i=1}^{n}(-1)^{n-i+1}\left(\partial_{n-i+1}^{1}(\mathbf{x})-\partial_{n-i+1}^{2}(\mathbf{x})\right)\right) \\
= & \partial_{n}^{S}(\mathbf{x})+(-1)^{j+n+1} \omega_{n-1}\left(\partial_{n}^{S}(\mathbf{x})\right) \in C_{n-1}(B ; \mathbb{Q})
\end{aligned}
$$

Note that

$$
\partial_{n}^{S}\left(\mathbf{x} \pm \omega_{n} \mathbf{x}\right)= \begin{cases}\partial_{n-1}^{S}(\mathbf{x}) \pm \omega_{n-1} \partial_{n-1}(\mathbf{x}) & n \text { odd } \\ \partial_{n-1}^{S}(\mathbf{x}) \mp \omega_{n-1} \partial_{n-1}(\mathbf{x}) & n \text { even }\end{cases}
$$

because of the $i \leftrightarrow n-i+1$ switch, which is why we need the $(-1)^{j}$ in the definition of $C_{n}^{S}(B, S ; \mathbb{Q})$.

Thus, we have a chain complex $C_{n}^{S}(B, S ; \mathbb{Q})$ with rational coefficients and boundary maps $\partial_{n}^{S}$; passing to the dual gives a cochain complex $C_{S}^{n}(B, S ; \mathbb{Q})$ with coboundary maps $\delta_{S}^{n}$.

Definition 5.7. The homology and cohomology spaces

$$
H_{n}^{S}(B, S ; \mathbb{Q})=\operatorname{ker}\left(\partial_{n}^{S}\right) / \operatorname{Im}\left(\partial_{n+1}^{S}\right), \quad H_{S}^{n}(B, S ; \mathbb{Q})=\operatorname{ker}\left(\delta_{S}^{n}\right) / \operatorname{Im}\left(\delta_{S}^{n-1}\right)
$$

of the chain complex $C_{n}^{S}(B, S ; \mathbb{Q})$ are the $S$-homology and $S$-cohomology of the virtual biquandle $(B, S)$.

Remark 5.8. We notice here that the $S$-chain complex is independent of the biquandle structure on $B$; it uses $B$ only as a set and does not actually use the biquandle operations. 
Armed with our S-homology theory, we can see what kind of function $v: B \times B \rightarrow$ $\mathbb{Q}$ must be to give the same contribution to the Boltzmann weight before and after a vIII move.
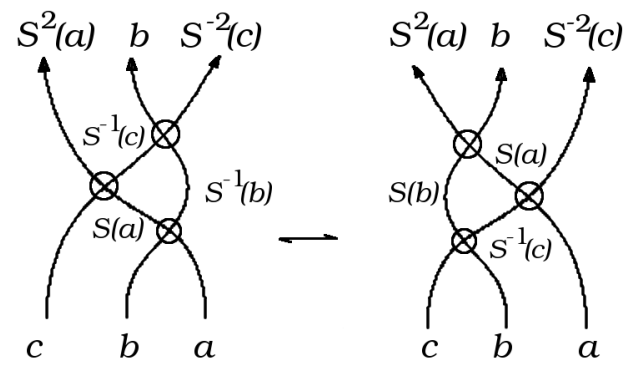

Using our counting rule, the contribution to the Boltzmann weight from the diagram on the left is

$$
\begin{gathered}
c_{l}=v(a, b)-v\left(S^{-1}(b), S(a)\right)+v(S(a), c)-v\left(S^{-1}(c), S^{2}(a)\right) \\
+v\left(S^{-1}(b), S^{-1}(c)\right)-v\left(S^{-2}(c), b\right)
\end{gathered}
$$

while the contribution from the diagram on the right is

$$
\begin{gathered}
c_{r}=v(b, c)-v\left(S^{-1}(c), S(b)\right)+v\left(a, S^{-1}(c)\right)-v\left(S^{-2}(c), S(a)\right) \\
+v(S(a), S(b))-v\left(b, S^{2}(a)\right) .
\end{gathered}
$$

Note that

$$
\begin{aligned}
\partial_{3}^{S}\left((a, b, c)-\left(S^{-2}(c), b, S^{2}(a)\right)\right)= & -(b, c)+\left(S^{-1}(b), S^{-1}(c)\right)+\left(b, S^{2}(a)\right) \\
& -\left(S^{-1}(b), S(a)\right)+(S(a), c)-\left(a, S^{-1}(c)\right) \\
& -\left(S^{-1}(c), S^{2}(a)\right)+\left(S^{-2}(c), S(a)\right)+(a, b) \\
& -(S(a), S(b))+\left(S^{-1}(c), S(b)\right)-\left(S^{-2}(c), b\right)
\end{aligned}
$$

so that $c_{l}-c_{r}=0$ if and only if $v$ evaluates to 0 on $\partial_{3}^{S}(z)$ for all $z \in C_{3}^{S}(B, S ; \mathbb{Q})$. That is, the condition we need for the contribution to the Boltzmann weight from $v$ to be invariant under $v I I I$ moves using our counting rule is simply that $v \in$ $C_{S}^{2}(B, S ; \mathbb{Q})$. Note that Lemma 2.4 implies that the other oriented virtual type III moves are equivalent to the one depicted here.

We are almost ready to define our virtual cocycle invariants. The final step comes from the last oriented virtual move, the mixed move $v$. It is here that we get interaction between the classical and virtual cocycles.

Lemma 2.4 and figure 5 of 3 show that in the presence of the oriented classical and virtual type II moves, a mixed move with one classical crossing is equivalent to (1) the same move with the classical crossing type switched and to (2) the same move with the orientation of the virtual strand reversed. Thus, all eight possible oriented mixed moves are equivalent, and it is sufficient to consider only the one move pictured in Proposition 5.10.

Definition 5.9. Let $(B, S)$ be a virtual biquandle. Then a classical cocycle $\phi \in$ $C_{Y B}^{2}(B ; \mathbb{Q})$ is compatible with an $S$ cocycle $v \in C_{S}^{2}(B, S ; \mathbb{Q})$ if for all $a, b, c \in B$ we 
have

$$
\begin{aligned}
\phi(a, b)-\phi(S(a), S(b))= & v(b, c)+v\left(a, S^{-1}(c)\right)+v\left(S^{-1}(c), S\left(a^{b}\right)\right) \\
& +v\left(S^{-2}(c), S\left(b_{a}\right)\right)-v\left(a^{b}, c\right)-v\left(b_{a}, S^{-1}(c)\right) \\
& -v\left(S^{-1}(c), S(b)\right)-v\left(S^{-2}(c), S(a)\right) .
\end{aligned}
$$

If we further have $\phi(a, b)-\phi(S(a), S(b))=0$ for all $a, b \in B$, we say that $\phi$ and $v$ are strongly compatible.

Note that $v$ and $\phi$ are strongly compatible if and only if both are compatible with the zero cocycle.

Proposition 5.10. If $v \in C_{S}^{2}(B, S ; \mathbb{Q})$ and $\phi \in C_{Y B}^{2}(B ; \mathbb{Q})$ are compatible, then the contributions to the Boltzmann weight of a virtual biquandle-colored link diagram before and after a mixed $v$ move are the same.

Proof. We simply read the condition off the picture:
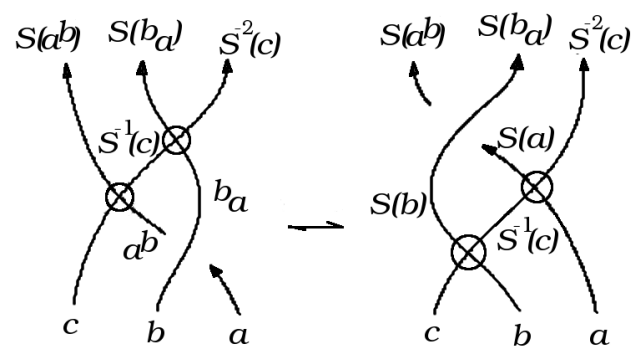

Definition 5.11. Let $(T, S)$ be a finite virtual biquandle, $L$ a virtual link diagram, $\phi$ a reduced Yang-Baxter 2-cocycle of $B$ and $v$ an $S$ 2-cocycle of $(T, S)$ compatible with $\phi$. For each $f \in \operatorname{Hom}(V B(L),(T, S))$ considered as a coloring $f(L)$ of $L$, let

$$
B W_{c}(f)=\sum_{+ \text {crossing }} \phi(u, o)-\sum_{- \text {crossing }} \phi(u, o)
$$

and let

$$
B W_{v}(f)=\sum_{\text {virtual crossing }} v(r, l)-v\left(S^{-1}(l), S(r)\right)
$$

where $u, o$ denote the under-crossing and over-crossing colors at classical crossings (inbound for positive crossings, outbound for negative) and $r, l$ denote the right and left inbound colors at a virtual crossing. Then the virtual Yang-Baxter 2-cocycle invariant is the set with multiplicities

$$
\Phi_{v Y B}(L,(T, S), \phi, v)=\left\{B W_{c}(f)+B W_{v}(f) \mid f \in \operatorname{Hom}(V B(L),(T, S))\right\} .
$$

Alternatively, we can convert the invariant into a Laurent polynomial (clearing denominators in $\phi$ and $v$ by scalar multiplication if necessary) to obtain

$$
\Phi_{v Y B}=\sum_{f \in \operatorname{Hom}(V B(L),(T, S))} t^{B W_{c}(f)+B W_{v}(f)} .
$$

We will frequently omit the $L,(T, S), \phi$ and $v$ from the notation when these are clear from the context. 
If $\phi$ and $v$ are strongly compatible, the contributions from $v$ and $\phi$ are independent and we can sharpen the invariant to obtain a two-variable Laurent polynomial

$$
\Phi_{v Y B}=\sum_{f \in \operatorname{Hom}(V B(L),(T, S))} t^{B W_{c}(f)} s^{B W_{v}(f)}
$$

or a multiset

$$
\Phi_{v Y B}=\left\{\left(B W_{c}(f), B W_{v}(f)\right) \mid f \in \operatorname{Hom}(V B(L),(T, S))\right\} .
$$

By construction, $\Phi_{v Y B}$ is an invariant of virtual isotopy. Curiously, unlike in the classical case, coboundaries can contribute non-trivially to the Boltzmann weight for a given coloring. More specifically, as in the classical case, a virtual coboundary breaks down into weights on semiarc endpoints, i.e.

$$
v(x, y)-v\left(S^{-1}(y), S(x)\right)=2 f(x)+2 f(y)-2 f(S(x))-2 f\left(S^{-1}(y)\right),
$$

but even if both the classical and virtual cocycles are coboundaries, the weights do not cancel as in the classical case unless we have $\phi=\delta_{Y B}^{1}(f)$ and $\frac{1}{2} v=\delta_{S}^{1}(f)$ for the same $f: B \rightarrow \mathbb{Q}$. Thus, cohomologous cocycles from either complex do not necessarily define the same virtual invariant.

Definition 5.12. Let $C_{D}^{2}\left(B, S^{\prime} \mathbb{Q}\right)$ be the subspace of $C_{S}^{2}(B, S ; \mathbb{Q})$ generated by cocycles of the form

$$
\chi_{(a, b)}+\chi_{\left(S^{-1}(b), S(a)\right)} .
$$

Elements of $C_{D}^{2}(B, S ; \mathbb{Q})$ will be called degenerate 2-cochains.

If $v \in C_{D}^{2}(B, S ; \mathbb{Q})$, then $v$ always contributes zero to the Boltzmann weight. In particular, if two $S 2$-cocycles $v, v^{\prime}$ differ by an element of $C_{D}^{2}(B, S ; \mathbb{Q}) \cap C_{S}^{2}(B, S ; \mathbb{Q})$, then for every compatible classical cocycle $\phi$, the pair $(\phi, v)$ defines the same invariant as $\left(\phi, v^{\prime}\right)$.

We close this section with a few observations. First, for every classical biquandle $\left(T, S=\operatorname{Id}_{B}\right)$, the zero virtual cocycle $v=0$ is compatible with every reduced YangBaxter cocycle $\phi$; this case is the classical Yang-Baxter cocycle invariant. Secondly, evaluating the polynomial version of the invariant at $t=1$ ( or $t=s=1$ in the strong case) yields the virtual biquandle counting invariant $|\operatorname{Hom}(V B(L),(T, S))|$. Moreover, in the strong case, setting $s=t$ yields the single-variable version of $\Phi_{v Y B}$. Lastly, if $v$ and $\phi$ are strongly compatible, then the presence of any nonzero power of $s$ in the two-variable version of the invariant indicates that the link in question is non-classical, since a classical link will always have a total contribution of 0 from virtual crossings.

\section{VIRTUAL 2-COCYCLE INVARIANT EXAMPLES}

In this section we present some examples of virtual Yang-Baxter 2-cocycle invariants. These were computed using Maple with programs available for download in the text files virtualbiquandles-maple.txt, biquandles-maple.txt, and yangbaxtercohomology.txt, at www.esotericka.org/quandles.

Our Maple notation uses Gaussian integers for crossing labels in virtual signed Gauss codes with imaginary part 0 for positive crossings, imaginary part $\pm i$ for 
negative crossings and imaginary part $\pm 2 i$ for virtual crossings. The absolute value of the real part of the label gives the crossing label; we use negative entries for under-crossings and right-hand virtual labels, and positive entries for over-crossings and left-hand virtual labels. A zero entry separates components. For example, the virtual signed Gauss code $\mathrm{U} 1^{-} \mathrm{R} 2 \mathrm{O3}^{+} \mathrm{O} 1^{-} \mathrm{L} 2 \mathrm{U} 3^{+}$in our Maple notation becomes

$$
[-1-I,-2-2 * I, 3,1+I, 2+2 * I,-3,0] \text {. }
$$

The program vblist takes a biquandle matrix (actually, a 4-tuple of operation matrices) and computes the automorphism group, then outputs one representative of each conjugacy class, with a permutation $\sigma \in S_{n}$ represented by the vector $[\sigma(1), \ldots, \sigma(n)]$.

The program vbhomlist takes a virtual Gauss code, a biquandle matrix and a permutation $\mathrm{S}$ and computes the set of all colorings of the virtual link represented by a virtual Gauss code by the virtual biquandle. vcocycles finds a basis for the space of $S$ 2-cocycles given a permutation $S$. These 2 -cocycles are represented by $n^{2}$-component vectors where the $n(i-1)+j$ entry represents the coefficient of $\chi_{(i j)}$, where $n$ is the cardinality of $B$. ybcompatfind takes a biquandle matrix $\mathrm{A}$ and a permutation $\mathrm{S}$ and finds a list of pairs of compatible classical and non-degenerate $S$-cocycles. Finally, vbinv takes a virtual signed Gauss code g, a biquandle matrix A, a permutation $\mathrm{S}$, a classical cocycle vector phi and an $S$-cocycle vector v and computes the polynomial virtual Yang-Baxter invariant. The program checks for strong compatibility and automatically computes the two-variable invariant (with variables $s, t)$ if the cocycles are strongly compatible, or the one-variable invariant (with variable $\mathrm{T}$ ) if they are only weakly compatible.

Example 6.1. The trivial biquandle of cardinality $n, T_{n}=\{1,2, \ldots, n\}$ with $a^{b}=a^{\bar{b}}=a_{b}=a_{\bar{b}}=a$, has a reduced Yang-Baxter cocycles spanned by $\chi_{(i j)}$ where $i \neq j$. If $K$ is a single-component virtual link (i.e., a virtual knot), then the only classical biquandle colorings by $T_{n}$ are the constant colorings and the classical 2cocycle invariant is equal to the counting invariant. However, if we endow $T_{n}$ with a non-trivial automorphism $S$ (and any permutation will do since $\operatorname{Aut}\left(T_{n}\right)=S_{n}$ ), then a compatible classical cocycle can contribute non-trivially to the virtual YangBaxter cocycle invariant. Here the virtual trefoil has $\Phi_{v Y B}(K)=3 t$ with respect to the Yang-Baxter cocycle $\phi=\chi_{(13)}+\chi_{(21)}+\chi_{(23)}$ and the zero $S$-cocycle $v=0$ of $\left(T_{3},(123)\right)$.
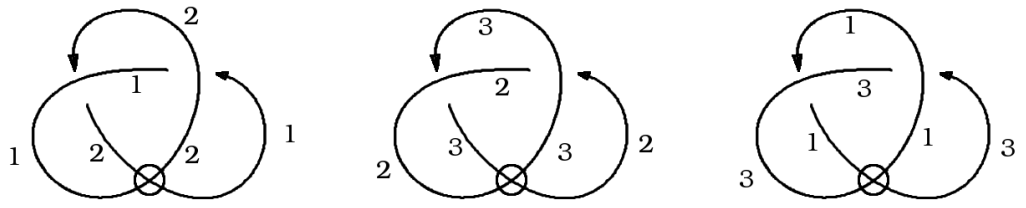

Example 6.2. The virtual biquandle $T=\{1,2,3\}, S=(23)$ with biquandle operation matrix below has $S$-cocycle $v=\chi_{(12)}+\chi_{(13)}$ strongly compatible with the zero classical cocycle $\phi=0$. The virtual link $L$ below has virtual Yang-Baxter 
cocycle invariant $\Phi_{v Y B}=2 s^{-2}+5+2 s^{2}$ with respect to this $\phi$ and $v$. Thus, this invariant detects the non-classicality of $L$. We list the colorings in the table.

$$
M_{T}=\left[\begin{array}{lll|lll}
1 & 1 & 1 & 1 & 1 & 1 \\
2 & 3 & 3 & 2 & 3 & 3 \\
3 & 2 & 2 & 3 & 2 & 2 \\
\hline 1 & 1 & 1 & 1 & 1 & 1 \\
3 & 3 & 3 & 3 & 3 & 3 \\
2 & 2 & 2 & 2 & 2 & 2
\end{array}\right]
$$

\begin{tabular}{|cccccccc|}
\hline$a$ & $b$ & $c$ & $d$ & $e$ & $f$ & $g$ & $h$ \\
\hline 1 & 1 & 1 & 1 & 1 & 1 & 1 & 1 \\
1 & 1 & 1 & 1 & 2 & 3 & 2 & 3 \\
1 & 1 & 1 & 1 & 3 & 2 & 3 & 2 \\
2 & 3 & 3 & 2 & 1 & 1 & 1 & 1 \\
2 & 3 & 2 & 3 & 2 & 3 & 2 & 3 \\
2 & 3 & 2 & 3 & 3 & 2 & 3 & 2 \\
3 & 2 & 2 & 3 & 1 & 1 & 1 & 1 \\
3 & 2 & 3 & 2 & 2 & 3 & 2 & 3 \\
3 & 2 & 3 & 2 & 3 & 2 & 3 & 2 \\
\hline
\end{tabular}

Example 6.3. Let $T=\mathbb{Z}_{3}$ with $a^{b}=a^{\bar{b}}=a_{b}=a_{\bar{b}}=2 a \bmod 3$. Then $T$ is an Alexander biquandle with $s=t=2$, and $\operatorname{Aut}(T)=\{(1),(12)\}$. $T$ has YangBaxter cocycle $\phi=\chi_{(13)}+\chi_{(23)}$, which is also an $S$-cocycle for the automorphism $S=(12) \in \operatorname{Aut}(T)$. Moreover, $\phi$ and $v=\phi$ are strongly compatible. The virtual link below has virtual Yang-Baxter invariant $\Phi_{v Y B}=5+2 s^{-2}+2 s^{2} t^{-2}$ with respect to $\phi=v$.

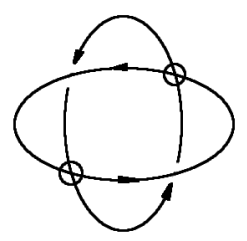

Example 6.4. The Alexander quandle $T=\mathbb{Z}_{6}=\{1,2,3,4,5,6\}$ with

$$
a^{b}=a^{\bar{b}}=5 a+2 b \bmod 6, \quad a_{b}=a_{\bar{b}}=a \bmod 6
$$

with $S=(26)(35)$ has an $S$-cocycle

$$
v=-\chi_{(14)}-2 \chi_{(16)}+2 \chi_{(22)}+2 \chi_{(23)}+\chi_{(24)}+2 \chi_{(25)}-\chi_{(34)}+\chi_{(42)}+\chi_{(43)}
$$

weakly compatible with the Yang-Baxter cocycle

$$
\phi=\chi_{(21)}+\chi_{(23)}+\chi_{(24)}+\chi_{(26)}-\chi_{(43)}-\chi_{(46)}-\chi_{(61)}-\chi_{(64)} .
$$

In fact, $\phi$ is a coboundary, but the virtual cocycle invariant defined by $\phi$ and $v$ includes non-zero contributions from $\phi$. For example, the virtual Hopf link below 
has invariant value $\Phi_{v Y B}=3 T^{-1}+6+3 T$; four of the twelve colorings have non-zero Boltzmann weight contributions from $\phi$.

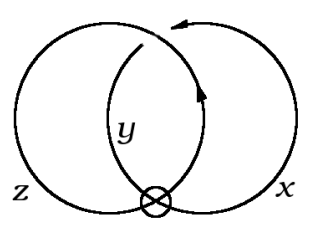

\begin{tabular}{|c|c|c|c|c|c|}
\hline$x$ & $y$ & $z$ & $x$ & $y$ & $z$ \\
\hline 1 & 1 & 1 & 1 & 1 & 4 \\
\hline 2 & 6 & 1 & 2 & 6 & 4 \\
\hline 3 & 5 & 1 & 3 & 5 & 4 \\
\hline 4 & 4 & 1 & 4 & 4 & 4 \\
\hline 5 & 3 & 1 & 5 & 3 & 4 \\
\hline 6 & 2 & 1 & 6 & 2 & 4 \\
\hline
\end{tabular}

\section{Questions For FUture RESEARCH}

We have only scratched the surface of the subject of virtual Yang-Baxter cocycle invariants. In this section we collect a few questions for future research.

As is well known, quandle cocycle invariants can be defined not only for knotted circles in $S^{3}$ and other 3-manifolds, but also for knotted surfaces in $\mathbb{R}^{4}$ or other 4-manifolds. The surface knot equivalents of virtual knots are known as abstract surface knots, equivalence classes of diagrams obtained by gluing together boxes containing triple points, pairs of crossed sheets, etc., under the equivalence relation defined by the Roseman moves. See [11. What modifications to our scheme are necessary to define virtual cocycle invariants for abstract surface knots?

The fact that we have two separate homology theories contributing to our invariants seems unsatisfying, especially as the compatibility condition is not expressed in terms of either coboundary map. Moreover, the fact that cohomologous cocycles do not define the same invariant seems troubling, as does the fact that the degeneracy condition for $S$ 2-cocycles is not just the coboundary condition. Perhaps there is a deeper cohomology theory which unifies both the classical Yang-Baxter and $S$-homology theories? It is because we suspect that such a theory awaits uncovering that we chose the term " $S$-homology", preferring to reserve the term "virtual biquandle homology" for this deeper theory.

Other counting rules for contributions to the Boltzmann weight from both classical and virtual crossings are possible, of course, and choosing different rules may result in different theories, defining potentially different invariants. We chose our rule for counting contributions from virtual crossings because it handles the $v I$ and $v I I$ moves easily; different rules might have other advantages and disadvantages. It is worth noting that the obvious alternative counting rules appear to suffer from the same deficiencies as the rule we ultimately chose, and most have the added disadvantage of requiring quotients to handle $v I$ and $v I I$ moves. For example, if we count just $v(r, l)$ instead of $v(r, l)-v\left(S^{-1}(l), S(r)\right)$, then we can use the same boundary map $\partial_{n}^{S}$, but two levels of quotients are required to handle the $v I$ and $v I I$ moves.

The virtual biquandle definition we used is the simpler of the two given in [14; instead of using the unary operations $S$ and $S^{-1}$ at virtual crossings, one can define a pair of binary operations at a virtual crossing, so that the virtual biquandle structure consists of six binary operations. A theory of cocycle invariants associated to this presumably richer virtual biquandle structure should prove interesting as well. 


\section{REFERENCES}

1. N. Andruskiewitsch and M. Graña. From racks to pointed Hopf algebras. Adv. Math. 178 (2003) 177-243. MR1994219 (2004i:16046)

2. J. S. Carter, A. S. Crans, M. Elhamdadi and M. Saito. Cohomology of Categorical SelfDistributivity. arXiv:math/0607417

3. J. S. Carter, D. Jelsovsky, S. Kamada, L. Langford and M. Saito. Quandle cohomology and state-sum invariants of knotted curves and surfaces. Trans. Amer. Math. Soc. 355 (2003) 3947-3989. MR 1990571 (2005b:57048)

4. J. S. Carter, M. Elhamdadi, and M. Saito. Twisted quandle homology theory and cocycle knot invariants. Algebr. Geom. Topol. 2 (2002) 95-135. MR1885217(2003a:57019)

5. J. S. Carter, M. Elhamdadi and M. Saito. Homology Theory for the Set-Theoretic Yang-Baxter Equation and Knot Invariants from Generalizations of Quandles. MR2128041 (2005k:57009)

6. J. S. Carter and M. Saito. Set-Theoretic Yang-Baxter Solutions via Fox Calculus. J. Knot Theory Ramifications 15 (2006) 949-956. MR2275090

7. C. Creel and S. Nelson, Symbolic computation with finite biquandles. J. Symbolic Comput. 42 (2007) 992-1000. MR.2361675

8. R. Fenn, M. Jordan-Santana and L. Kauffman. Biquandles and virtual links. Topology Appl. 145 (2004) 157-175. MR2100870(2005h:57015)

9. D. Hrencecin and L.H. Kauffman. Biquandles for virtual knots. J. Knot Theory Ramifications 16 (2007) 1361-1382. MR 2384830

10. N. Jackson. Extensions of racks and quandles. Homology, Homotopy Appl. 7 (2005) 151-167. MR 2155522 (2006f:18006)

11. N. Kamada and S. Kamada. Abstract link diagrams and virtual knots. J. Knot Theory Ramifications 9 (2000) 93-106. MR.1749502 (2001h:57007)

12. L. Kauffman. Virtual Knot Theory. European J. Combin. 20 (1999) 663-690. MR1721925 (2000i:57011)

13. L. H. Kauffman and D. Radford. Bi-oriented quantum algebras, and a generalized Alexander polynomial for virtual links. Contemp. Math. 318 (2003) 113-140. MR.1973514(2004c:57013)

14. L. H. Kauffman and V. O. Manturov. Virtual biquandles. Fundam. Math. 188 (2005) 103-146. MR 2191942(2006k:57015)

15. S. Nelson and J. Rische. On Bilinear Biquandles. Colloq. Math. 112 (2008), 279-289.

16. S. Nelson and J. Vo. Matrices and Finite Biquandles. Homology, Homotopy Appl. 8 (2006) 51-73. MR 2246021(2007j:57009)

Department of Mathematics, Whittier College, 13406 Philadelphia, P.O. Box 634, Whittier, California 90608-0634

E-mail address: jcenicer@poets.whittier.edu

Department of Mathematics, Claremont McKenna College, 850 Columbia Avenue, Claremont, California 91711

E-mail address: knots@esotericka.org 